\title{
REVIEW
}

\section{Emergency analgesia in the paediatric population. Part IV Paediatric sedation in the accident and emergency department: pros and cons}

\section{E Doyle}

This is the last in a series of four papers on emergency analegesia in the paediatric population. It discusses the use of paediatric sedation in the accident and emergency department.

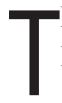
here is currently increased interest in the safe provision of sedation to children for minor surgical procedures, endoscopy, and radiological procedures. There are changing perceptions of what is acceptable in the restraint of children during painful procedures and increasing expectations of analgesia and amnesia from children, parents, nursing, and medical staff. The alternative to sedation is the use of general anaesthesia. There are increasing demands on the anaesthetic departments of most hospitals and the specialty is understaffed to meet the demands made upon it. ${ }^{1}$ These factors mean that the provision of general anaesthesia is often difficult and usually entails considerable delay and inconvenience for patients and families. The advantages and disadvantages of sedation compared with anaesthesia need to be considered.

There are problems with a clear definition of sedation being accepted by all concerned within a hospital. Sedation refers to a continuum of effects between mild anxiolysis and general anaesthesia. A state of light or conscious sedation is generally accepted and refers to a state of pharmacologically induced depression of the central nervous system where the airway is maintained, verbal contact (if appropriate) can be maintained with the patient and the patient is roused by gentle physical stimulation. There is usually a caution that the drugs and doses used should carry a wide enough margin of safety to render unintentional loss of consciousness unlikely. ${ }^{2}$ This state is often completely inadequate for the performance of a noxious procedure in a child. Some then accept a state of deep sedation where verbal contact with the patient is lost, the patient may not always maintain a clear airway, and may not respond to gentle physical stimulation. The difficulty comes because to many this state of deep sedation is equivalent to general anaesthesia in its effects and potential dangers. The General Dental Council, ${ }^{2}$ the Royal Colleges of Anaesthetists and Radiologists, ${ }^{3}$ the Royal College of Surgeons of England, ${ }^{4}$ and the British Society of Gastroenterology ${ }^{5}$ have produced guidelines on the safe use of sedation and all have refused to recognise a distinction between deep sedation and anaesthesia. The American Academy of Pedi- atrics does accept such a distinction and does not require an anaesthetist to be present during deep sedation but emphasises that deep sedation and anaesthesia are virtually inseparable for the purposes of monitoring. ${ }^{6}$ Deep sedation has been advocated as the desirable state for a sedated child as many investigations and procedures are impossible under conscious sedation. ${ }^{78}$ In terms of patient safety the issue is whether clinicians giving intravenous sedation can manage the potential complications especially airway obstruction and hypoventilation. Clinicians who administer intravenous sedation must be competent at managing an anaesthetised patient.

The requirements of a sedation regimen in children undergoing noxious procedures could be listed as rapid onset, adequate depth of sedation to allow for stimulation of the child during the procedure, the maintenance of spontaneous respiration, immobility, rapid recovery, and minimal side effects. Anaesthesia guarantees a still patient and good operating conditions. Modern anaesthetic drugs used in these circumstances have rapid elimination and predictable recovery characteristics associated with a rapid return to the pre-anaesthetic state and fitness for discharge from hospital. There is no time limit on the procedure imposed by the sedative technique or patient tolerance and as there is no delay in effect after administration or failure to anaesthetise the throughput of patients in a session is predictable and efficient.

Common problems with the traditional sedatives agents used in paediatric practice include failure to sedate adequately for the procedure and a prolonged period of sedation afterwards because of the production of active metabolites and the prolonged elimination of some drugs. This applies to trimeprazine, chloral hydrate, diazepam, and ketamine. Ketamine may cause hallucinations and nightmares. Cocktails of drugs are associated with a high incidence of side effects and prolonged sedation. ${ }^{9}$ Several series in which anaesthetic induction agents were used as sedative agents in children resulted in a significant incidence of hypoxic events. ${ }^{10-12}$ Midazolam seems to be the best of the currently available options. There is successful sedation in about $70 \%$ of children after $0.5 \mathrm{mg} / \mathrm{kg}$ by mouth although this refers to the state of conscious rather than deep sedation. ${ }^{13-16}$ It requires $30-45$ minutes after oral administration to be effective ${ }^{16}$ and has a predictable duration of action of 30-60 minutes. ${ }^{17}$ The pharmacokinetics are known and there is rapid elimination with an elimination half life of one to two hours ${ }^{18} 19$ and no active metabolites. It is also 
readily antagonised by flumazenil. Intravenous midazolam may be used alone shortly before a procedure or in modest doses to enhance the effect of an oral dose during the procedure. Intravenous use is associated with almost immediate effect and a similar rapid predictable recovery in the majority of children. ${ }^{20}$

In accident and emergency (A\&E) departments most interventions are likely to be painful and analgesia as well as sedation may be required. Most sedative agents including the benzodiazepines have no analgesic action and attempting to provide analgesia with these alone is likely to result in deep sedation or anaesthesia and techniques where analgesia is combined with sedation are required. This may take the form of a local anaesthetic technique performed with the aid of sedation or intravenous opioid given in an appropriate dose bearing in mind the synergism of sedative drugs and opioids with regard to hypoventilation. Entonox may have a place in providing a combination of sedation and analgesia either used alone or in combination with oral or intravenous drugs. Contraindications to its use must be sought and appropriate training of staff is required. The analgesic effects of entonox cease when its administration stops. If pain after the procedure is likely then some form of longer lasting regional or systemic analgesia should be administered in anticipation of this.

The safe and effective use of midazolam or ketamine for sedation in children undergoing procedures in A\&E departments has been described. In general not all children are adequately sedated and in some there is disinhibition or lack of cooperation during the procedure..$^{21}$

Whatever methods or drugs are used to sedate children there is a failure rate. Individuals have varying responses to the same dose of sedative and because of the requirement to err on the side of caution in dosing a significant minority of children will not be adequately sedated with sensible drug dosing and a small number may demonstrate paradoxical hyperexcitement or disinhibition. These problems occur particularly when patients are in pain..$^{23}$ The use of a combination of midazolam and pethidine in doses that exceeded most recommendations (up to $0.75 \mathrm{mg} / \mathrm{kg}$ of intravenous midazolam and $2.5 \mathrm{mg} / \mathrm{kg}$ of intravenous pethidine) and that were designed to produce a state of deep sedation for upper and lower gastrointestinal endoscopy had a failure rate of 3\%, an unsatisfactory degree of sedation that did permit endoscopy in $27 \%$ and a satisfactory outcome in $70 \%$. There was mild hypoxia in $14 \%$ of patients. This emphasises the variability between patients resulting in an unpredictable effect even when generous dosing regimens are used. ${ }^{24} \mathrm{~A}$ technique that has no failure rate is likely to entail excessive dosing for some children and the production of a state equivalent to general anaesthesia in a minority. The possibility of unsuccessful sedation should be made clear to the parents before the procedure. Any system developed for the management of paediatric sedation should accept a significant failure rate and a figure of $10 \%-20 \%$ has been suggested as reasonable. ${ }^{25}$

The safety record of general anesthesia in healthy children for investigations or minor surgical procedures is impressive and if sedation rather than anaesthesia is to be used for these procedures then it should have the same standard of safety with minimal morbidity and mortality in the order of $1 / 200000$. Some of the reasons for the safety of general anaesthesia under these circumstances bear examination. A child undergoing general anaesthesia will undergo a medical clerking and a personal or family history of anaesthetic problems will be elicited. The cardiovascular and respiratory systems will be examined and baseline observations made. Before anaesthesia the child will be assessed by an anaesthetist and whenever possible fasted for an appropriate length of time. The anaesthetist will tend to err on the side of caution if he or she is a trainee or a non-paediatric anaesthetist and decline to anaesthetise complex or potentially difficult patients. Anaesthesia will be administered with the help of a trained assistant in a dedicated area equipped to deal with most of the possible complications and the anaesthetist will be familiar with appropriate protocols for the initial management of rare but potentially disastrous complications of anaesthesia. Cardiovascular and respiratory monitoring equipment will be applied before or shortly after induction and left in place until the child has recovered from anaesthesia. Some form of active airway management will be performed. The anaesthetist will remain with the child for the duration of the procedure and react to unacceptable deviations in airway, ventilation or cardiovascular parameters. Postoperatively the child will remain in a recovery room with one to one nursing from an appropriately trained nurse until they fulfil explicit documented criteria for return to a ward. The child will usually be kept in hospital for one to two hours and again fulfil explicit documented criteria before discharge. If sedation is to match this standard of safety then there must also be assessment of patients before sedation, the exclusion of potentially high risk cases, the use of appropriate monitoring equipment and personnel dedicated to the care of the patient during and after the procedure.

There is evidence that current practice in many hospitals when sedating children would not stand up to dispassionate scrutiny in the event of an adverse outcome. In a survey of current practice in 38 hospitals in Scotland when sedating children only three had a formal protocol for paediatric sedation, in one third of hospitals patients were not fasted before the procedure, in nearly $20 \%$ no monitoring of any kind was used. There were no deaths but several critical incidents were reported. ${ }^{26}$ of 32 emergency rooms that undertook sedation of children in North America, $40 \%$ did not have fasting guidelines and in those with guidelines they were not strictly enforced. ${ }^{27}$ In the United States an assessment of adverse drug reports submitted to the FDA between 1967 and 1994 and a survey revealed at least 52 deaths and 27 episodes of serious morbidity including six episodes of permanent neurological damage and 15 prolonged hospitalisations attributed to sedation. The causes of these events were mainly drug overdose, inadequate monitoring, inadequate training of the personnel involved, or premature discharge. ${ }^{28} \mathrm{~A}$ prospective assessment of the complications of sedation and general anaesthesia in 424 children for computed tomography or magnetic resonance imaging demonstrated a significantly higher incidence of adverse effects in the group receiving sedation than in those given general anaesthesia. These included inadequate sedation (16\%), hypoxia $(2.9 \%)$, drug reactions $(2 \%)$, and other problems (1.8\%). Of 376 attempted procedures $6 \%$ were unsuccessful. There was one adverse event in the general anaesthetic group related to failure of an item of equipment. ${ }^{29}$ A prospective comparison of the incidence of hypoxic episodes during upper gastrointestinal endoscopy in healthy children with either sedation or anaesthesia demonstrated an incidence of $89 \%$ for mild hypoxia $\left(\mathrm{S}_{\mathrm{p}} \mathrm{O}_{2}<95 \%\right)$ and $50 \%$ for severe hypoxia $\left(\mathrm{S}_{\mathrm{p}} \mathrm{O}_{2}<90 \%\right)$ in the group given sedation compared with incidences of $5 \%$ and zero respectively in the anaesthetised patients. ${ }^{30}$ Although these are not life threatening complications they provoke thought and there is cause for concern about the relative risks of sedation compared with anaesthesia in children. It is important to bear in mind here the concept of statistical power. A trouble free series of several hundred sedations does not demonstrate equivalent safety to general anaesthesia if a serious adverse event occurs only once in several thousand cases whichever technique is used.

Groups who are likely to present problems during sedation are largely predictable and include neonates and children aged less than 6 months, those with renal or hepatic impairment, respiratory distress, abnormalities of the airway, CNS disease, and patients on multiple drug therapies. Given the potential risk of regurgitation and aspiration during inadvertent deep 
sedation all candidates for sedation should be fasted in the same way as children undergoing a general anaesthetic. Toddlers and infants are often more difficult to sedate adequately than older children. These are also the children who will quickly become severely hypoxic in the event of airway obstruction as they have a higher oxygen consumption than older children. Small children are also less amenable to distraction, parental separation, and counselling than older children and venous access can be difficult. It may be appropriate for a sedation policy to specifically exclude children below a certain age and 5 years may be a reasonable cut off. The drugs given should be recorded. Observation of the child and of the monitor(s) should be performed by someone with this as their only responsibility. This should continue until the child meets criteria for adequate recovery from sedation. Monitoring of the child should entail a minimum of continuous pulse oximetry while breathing air as a monitor of hypoventilation and some assessment of the degree of sedation. The intermittent recording of respiratory rate has been shown to be a late and insensitive monitor of hypoventilation ${ }^{31}{ }^{32}$ and this cannot be relied upon to give an early warning of this problem. The use of pulse oximetry while breathing air is a more sensitive monitor of hypoventilation ${ }^{33}$ and in the absence of other causes of hypoxia such as impaired gas exchange, mild hypoxia (arterial oxygen saturation $<94 \%$ ) indicates hypoventilation. The observed values should be recorded for on a time based anaesthetic type record for audit and medicolegal purposes.

In North America and Europe anaesthesia and sedation in appropriate patients are often administered by trained nurses $^{34}$ and, given the difficulty in obtaining anaesthetic time for many departments, there would seem to be scope for the development of this role in the administration and monitoring of sedative drugs to children. Sedation by nurses is currently used in a small number of paediatric hospitals in the UK to provide sedation of children for radiological investigations. Nurses can assess patients according to an agreed protocol, prescribe and administer drugs, monitor the effects of this during a procedure, and assess the patient before discharge. Nurses tend to be more permanent members of staff than trainee doctors in A\&E departments and the investment in training them could be recouped over time. The role of play specialists during procedures under sedation could probably also be expanded with benefit. The role of distraction and play therapy during the induction of anaesthesia, procedures performed under sedation in operating theatres, and in chronically ill children undergoing repeated noxious interventions is well described. A comprehensive approach to providing sedation for noxious procedures should include expertise of this type. The question of parental presence during the procedure should also be considered in departmental protocols. If a child is not anaesthetised then there is likely to be a wish from many parents to remain with the child during the procedure and this may or may not be considered acceptable by the department. The medical and nursing staff involved in giving sedation to children will require adequate training. Issues that need to be considered and incorporated in an audit system are whether the staff involved should have a current PALS provider certificate, how frequently staff should perform sedation to remain competent, and the frequency of mandatory updates on paediatric sedation and resuscitation.

A successful programme of sedation in children will probably require

- acceptance that this is not necessarily an easier or cheaper option than general anaesthesia

- commitment from senior doctors and nurses within the department

- the production of protocols with indications for sedation, contraindications, fasting requirements, a consent form, maximum drug doses, monitoring requirements, appropriate treatment of problems during sedation, and discharge criteria

- the possible exclusion of children below a certain age

- the provision of dedicated time in the form of an "operating list" with adequate medical, nursing, and other staffing

- an expectation of a significant number of failures that are referred for the procedure under general anesthesia

- adequate monitoring equipment and recovery facilities

- an effective audit system.

With the investment of considerable time and effort a department should be able to ensure that procedures are performed in children under sedation with a standard of safety that which is similar to general anaesthesia. The main drawback in a well organised system should be a significant failure rate that requires subsequent general anaesthesia for the child.

\section{SUMMARY}

Children and infants exposed to a noxious stimulus feel pain to the same extent as adults. For a child, the unfamiliar and frightening environment of the A\&E department can compound and heighten their experience of pain. Provision of anlagesia to children in A\&E is currently often inadequate because of poor pain recognition and assessment and staff inexperience.

In the four papers in our series on paediatric pain relief we have discussed several ways of improving paediatric pain relief in the A\&E department. Children's pain shoul be assessed and quantified using objective pain relief. Effective analgesic agents should be given in adequate dose and appropriate route for the type and level of pain suffered. Physical methods of pain relief can be used such as splints and dressings. Psychological methods of pain relief and anxiolysis range from play therapy and guided imagery to providing a department decorated in a "child friendly" style, with children seen in an area audio-visually separate from adult patients where possible. Conscious sedation can be effectively used to improve a child's experience of unpleasant procedures.

By tackling these issues we can relieve pain and ultimately improve the experience of children attending our A\&E departments.

\section{REFERENCES}

1 Audit Commission. Anaesthesia under examination. London: Audit Commission, 1997

2 General Dental Council. Report of an expert working party of the Standing Dental Advisory Committee. General Anaesthesia, sedation and resuscitation in dentistry. (The Poswillo Report.) London: General Council, 1990.

3 Royal Colleges of Anaesthetists and Radiologists. Report of a Joint Working Party. Sedation and anaesthesia in radiology. London: Royal Colleges of Anaesthetists and Radiologists, 1992.

4 Royal College of Surgeons of England. Commission of the provision of surgical services. Report of the working party on guidelines for sedation by non-anaesthetists. London: Royal College of Surgeons, 1993.

5 Bell GD, McCloy RF, Charlton JE, et al. British Society of Gastroenterology: report of a working party of the Endoscopy Committee. Recommendations for standards of sedation and patient monitoring during gastrointestinal endoscopy. Gut 1991;32:823-7.

6 Guidelines for monitoring and management of pediatric patients during and after sedation for diagnostic or therapeutic procedures. Pediatrics 1992;89:1110-15

7 Spear RM. Deep sedation for radiological procedures in children: enough is enough. Paediatr Anaesth 1993;3:325-7.

8 Murphy MS. Sedation for invasive procedures in paediatrics. Arch Dis Child 1997;77:281-6.

9 Cote CJ. Sedation for the pediatric patient. Pediatr Clin North Am 1994;41:31-58.

10 Bloomfield EL, Masaryk TJ, Caplin A. Intravenous sedation for MR imaging of the brain and spine in children: pentobarbital versus propofol. Pediatr Radiol 1993;186:93-7.

11 Strain JD, Cambell JB, Harvey LA, et al. IV nembutal: safe sedation for children undergoing CT? Am J Radiol 1988;151:975-9. 
12 Frankville D, Spear R, Fisher B, et al. Is routine endotracheal intubation necessary when using intravenous anesthesia for MRI? Anesthesiology 19 92;77:A1165.

13 Jones RDM, Visram AR, Kornberg JP, et al. Premedication with oral midazolam in children - an assessment of psychomotor function, anxiolysis, sedation and pharmacokinetics. Anaesth Intensive Care 1994;22:539-44.

14 Parnis SJ, Foate JA, van der Walt JH, et al. Oral midazolam is an effective premedication for children having day-stay anaesthesia. Anaesth Intensive Care 1992;20:9-14.

15 Feld LH, Negus JB, White PF. Oral midazolam preanesthetic medication in pediatric outpatients. Anesthesiology 1990;73:831-4.

16 Weldon BC, Watcha MF, White PF. Oral midazolam in children: effect of time and adjunctive therapy. Anesth Anal 1992;75:51-5.

17 Ferguson S, Ball AJ. Sedation and sedative drugs in paediatrics. $\mathrm{Br} \mathrm{J}$ Hosp Med 1996;55:611-15.

18 Payne K, Mattheyser FJ, Liebenberg D, et al. The pharmacokinetics of midazolam in paediatric patients. Eur J Clin Pharmacol 1989:37:267-72.

19 Jones RDM Chan K, Roulson CJ, et al. Pharmacokinetics of flumazenil and midazolam. BrJ Anaesth 1993;70:286-92.

20 Cole WHJ. Midazolam in paediatric anaesthesia. Anaesth Intensive Care 1982;10:36-9

21 McGlone RG, Ranasinghe S, Durham S. An alternative to 'brutacaine': a comparison of low dose intramuscular ketamine with intranasal midazolam in children before suturing. J Accid Emerg Med 1998; 15:231-6.

22 Davies FC, Waters M. Oral midazolam for conscious sedation of children during minor procedures. J Accid Emerg Med 1998;15:244-8.

23 Illingworth KA, Simpson KH. Anaesthesia and analgesia in emergency medicine. 2nd edn. Oxford: Oxford University Press, 1998.
24 Sibal A, Davison S, Murphy MS. High-dose midazolam/meperidine sedation for pediatric endoscopy. Gastroenterology 1996; 110:A39.

25 Sury MRJ. The pros and cons of anaesthesia for children who need radiological procedures. Paediatr Anaesth 1993;3:329-31.

26 Morton NS, Oomen GJ. Towards safer sedation of children. Scottish Health Bulletin 1998;56:565-71.

27 Barnes SD, Sikavitsas DO. ER sedation practices: are they safe? Anesth Analg 1998;86:S158.

28 Cote CJ, Alderfer RJ, Notterman DA, et al. Sedation disasters: adverse drug reports in pediatrics - FDA, USP and others. Anesthesiology 1995;83:A1 182.

29 Malviya S, Voepel-Lewis T, Tait AR. Adverse events and risks of sedation and general anesthesia in children undergoing MRI and CT scans. Anesth Analg 1998;86:S410.

30 Dubreuil M, Da Conceicao, Lamireau T, et al. Oxygen desaturation in children during gastro intestinal endoscopy: general anaesthesia versus sedation. Anesthesiology 1995;83:A1 184.

31 Catley DM, Thornton C, Jordan J, et al. Pronounced episodic oxygen desaturation in the postoperative period: its association with ventilatory pattern and analgesic regimen. Anesthesiology 1985;63:20-8.

32 Kluger MT, Owen H, Watson D. Oxyhaemoglobin saturation following elective abdominal surgery in patients receiving continuous intravenous infusion or intramuscular morphine analgesia. Anaesthesia 1992;47:256-60.

33 Hutton $\mathbf{P}$, Clutton-Brock T. The benefits and piffalls of pulse oximetry. BM 1993;307:457-8.

34 Lin YC. Sedation of children for magnetic resonance imaging by nurses versus anaesthesiologists. Anesth Analg 1998;86:S409. 